\title{
MRI compatibility of position-sensitive photomultiplier depth-of-interaction PET detectors modules for in-line multimodality preclinical studies
}

\author{
J.J. Vaquero ${ }^{\mathrm{a}, \mathrm{b}, *}$, J.J. Sánchez ${ }^{\mathrm{b}}$, J.M. Udías ${ }^{\mathrm{c}}$, J. Cal-González ${ }^{\mathrm{c}}$, M. Desco ${ }^{\mathrm{a}, \mathrm{b}}$ \\ a Universidad Carlos III de Madrid, Departamento de Bioingeniería e Ingeniería Aeroespacial, Avda. de la Universidad 30 Leganés, 28911 Madrid, Spain \\ ${ }^{\mathrm{b}}$ Instituto de Investigación Sanitaria Gregorio Marañón, Madrid, Spain \\ c Grupo de Física Nuclear, Departamento de Física Atómica, Molecular y Nuclear, Universidad Complutense de Madrid, CEI Moncloa, Madrid, Spain
}

Keywords:

PET/MRI

Photomultiplier

Magnetic field

Preclinical imaging

Multimodality imaging

Molecular imaging

\begin{abstract}
This work addresses the feasibility of a small-animal, in-line PET/MR system based on PositionSensitive Photo Multiplier Tubes (PS-PMTs). To this end, we measured the effects of static magnetic fields on the PS-PMTs performance in order to explore the minimal tandem separation between the PET and MR subsystems to preserve their respective performances. We concluded that it is possible to achieve minimal degradation of the PET scanner performance (after a system recalibration) if the magnetic field strength influencing the PET detectors is less than $1 \mathrm{mT}$ and if it is oriented perpendicularly to the longitudinal axis of the tube. Therefore, we predict that it will be possible to maintain the PET image quality if it is placed outside the $1 \mathrm{mT}$ line.
\end{abstract}

\section{Introduction}

First publications about combined MRI/PET imagers date from 1997 and since then the number of designs has steadily grown [1-4]. Substantial efforts were focused on overcoming the interference between the two imaging systems when they are coaxially integrated in a single hybrid unit [4-16]. Other approaches avoided this close integration and proposed an in-line, tandem combination of the PET and the MRI systems with a revolving bed in between [17]. While this solution has the advantage that none of the imaging systems (the PET or the MRI) suffer performance degradation due to the proximity of the other one, the resulting device occupies a large space, requires a complex mechanism for patient transport and cannot acquire simultaneous PET/MR scans. On the contrary, space is not a mayor issue when using low-field, permanent based MRI preclinical imagers. In this case both systems can be closely attached since the MRI system fringe field can be considered negligible outside the magnet itself. However, the worse quality of the low-field MR images and the impossibility of doing simultaneous acquisitions are still two clear drawbacks for certain applications.

Our approach makes use of state-of-the-art commercial preclinical scanners: an MRI system (Bruker Biospec 70/20USR) and a small-animal PET (SEDECAL Argus PET/CT) [18], placing both

\footnotetext{
* Corresponding author at: Universidad Carlos III de Madrid, Departamento de Bioingeniería e Ingeniería Aeroespacial, Avda. de la Universidad 30, Leganés 28911, Madrid, Spain. Tel: +34914265067.
}

systems as close as possible to each other with their axial axes perfectly aligned. In this way it is possible to use a common, straightforward bed-moving mechanism to transport the animal between the two scanners. Simultaneous data acquisition is not possible, but the exquisite image quality of both systems is preserved. Since the static magnetic fringe field of the MRI system can affect the PS-PMT behavior [19], we evaluated the detector performance when operated under these conditions, as well as the potential degradation of final PET image quality.

\section{Materials and methods}

A test-bench was built using two Argus detector modules placed $12 \mathrm{~cm}$ apart and connected with a PET data acquisition system working in coincidence mode. The Argus detectors consist of a phoswich (two-layer scintillator with a $7 \mathrm{~mm}$ long LYSO and an $8 \mathrm{~mm}$ long GSO crystals, [20]) optically glued to a Hamamatsu R8520-00-C12 PS-PMT and with a signal pre-amplification board. The coincidence acquisition system is based on a 622 LeCroy's NIM logic unit and a $2 \times 6$-channel 12-bit ADC/TDC module (A\&D Precision Co., USA).

As a reference for performance assessment, field-flood images, phoswich diagrams and crystal spectra were acquired using a Plexiglas laminar container filled with a ${ }^{68} \mathrm{Ga}$ solution, with no magnetic field present (Fig. 1). The upper panel shows (from left to right) the field-flood illumination raw image, the phoswich diagram and the GSO spectra for all the crystals. Individual spectra for the LYSO and the GSO crystals of two phoswiches 

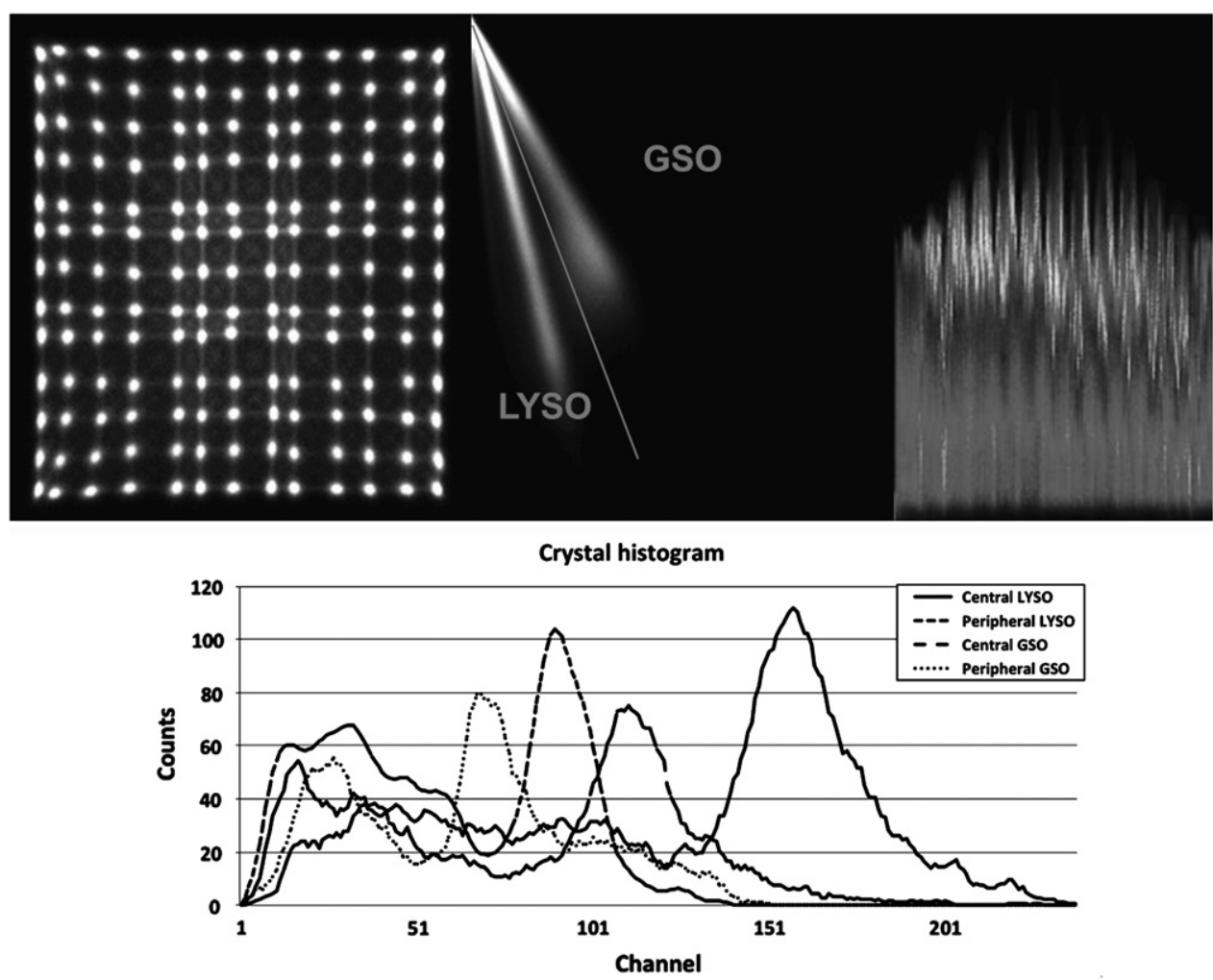

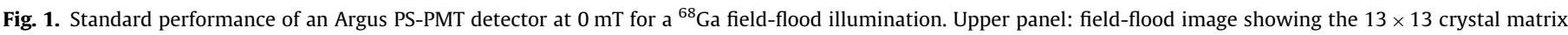

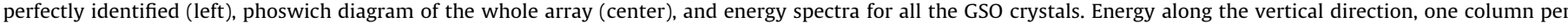
crystal (right). Bottom panel: individual spectra for the LYSO and the GSO crystals of two phoswiches arbitrarily selected at the center and the periphery.

arbitrarily selected at the center and the periphery are shown at the bottom panel.

A set of $\mathrm{NdFeB}+\mathrm{NiCuNi}$ magnets (6250 and $330 \mathrm{mT})$ was used to create a magnetic field similar to the one that would appear in the PET detector when placed nearby the MRI system in the tandem configuration. The magnetic field was tailored replicating the fringe field of the Bruker magnet (Fig. 2). Magnetic field intensity and orientation were adjusted by placing magnets at different distances along the three orthogonal directions defined by the PS-PMT axis (Fig. 3). Field values were confirmed using a tesla-meter with a Hall cell (Phywe 13610, Germany). We explored the effect of the magnetic field along three orthogonal spatial directions of the magnetic vector because the PS-PMT manufacturer describes different degradation of the device performance depending on the impinging direction of the magnetic field [19].

The detectors were exposed to different $\beta^{+}$sources: a Plexiglas laminar recipient filled with a ${ }^{68} \mathrm{Ga}$ solution for field-flood illuminations and a ${ }^{22} \mathrm{Na}$ point source for electronic adjustments. Images from those sources were created after acquiring data at a rate $<10 \mathrm{Kcps}$. These tests were repeated at five different field orientations with respect to the PS-PMT ( $X$ axis, $Y$ axis, $Z$ axis, XY and $\mathrm{XZ}$ axis), and at 1,3 , and $5 \mathrm{mT}$ intensities (Fig. 3).

Crystal and phoswich (DOI) energy spectra and look up tables (LUTs) as well as timing calibrations, sensitivity uniformity correction maps and energy resolution LUTs were generated. Count rate was registered for all the acquisitions to track possible sensitivity losses due to the exposure of the PS-PMT to the magnetic field.

\section{Results}

Intrinsic spatial resolution: Fig. 4 illustrates different examples of the crystal map degradation for different fringe field intensities

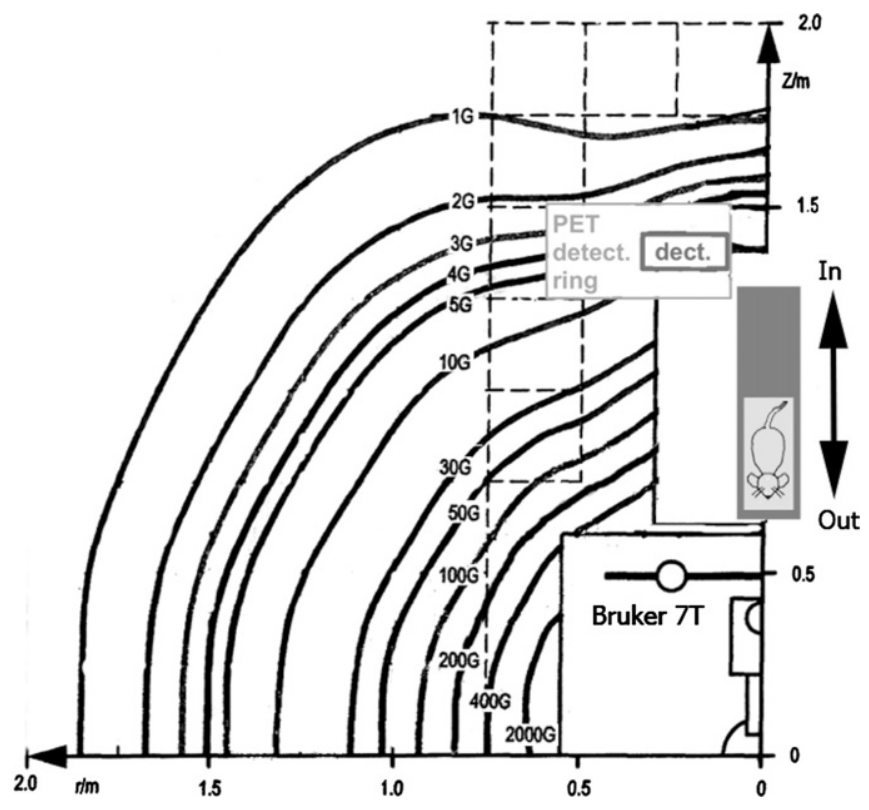

Fig. 2. Floor plan of the proposed tandem PET/MRI configuration. The axis origin is placed at the magnet center. The curved lines show the fringe magnetic field (in gauss). The PET detector is shown as a box with a smaller box representing the detector itself. A bed with a rat shows bed location and bed travel direction. Axis units are distance to the magnet center in meters.

and orientations. The upper panel shows raw images for the $\mathrm{XZ}$ axis and the bottom panel shows the crystals maps and the phoswich diagrams for the $Y$ axis. Field-flood images are distorted but mapping of the $13 \times 13$ phoswich crystals is still feasible for intensities lower than $3 \mathrm{mT}$, provided that only the $\mathrm{Y}$ axis component of the field is present. If a sizeable $\mathrm{X}$ component is 
present, the maximum magnetic field intensity that does not degrade the crystal map is as low as $1 \mathrm{mT}$. Phoswich diagrams are similarly affected.

Count rate: the PS-PMT manufacturer reports that in this kind of phototubes the electron trajectories of the secondary electrons emitted from the dynodes are affected by the external magnetic fields [19], decreasing the output signal of the tube, and therefore affecting the net count rate of the detector. Fig. 5 shows coincidence and singles count rates as a function of the external magnetic field to which the PS-PMT is exposed. The count rate

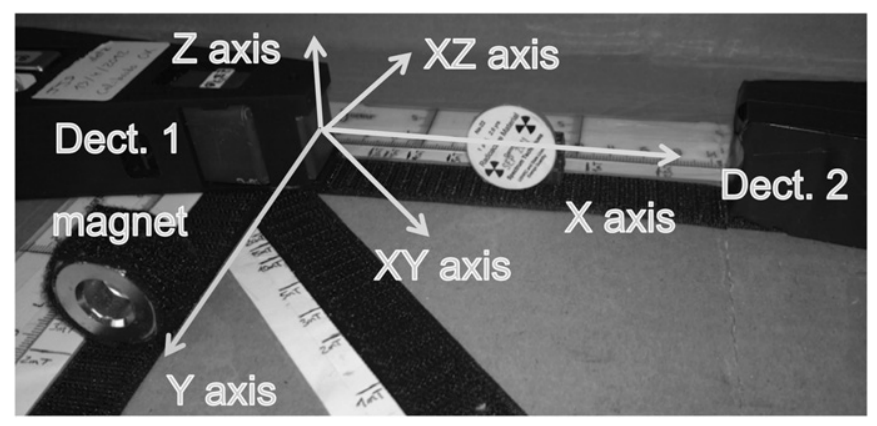

Fig. 3. Test-bench for performance evaluation of the PET detectors. The axes of the magnetic field are defined; a point source is shown placed in between the two detectors. One of the magnets used to replicate the fringe field is also shown. reduction observed in these plots depends on the lower number of events that produce a valid trigger due to the smaller signal output. This effect cannot be effectively compensated with a new adjustment of the trigger levels because the reduction of the tube output signal implies a smaller signal to noise ratio (SNR).

Energy resolution: Fig. 6 shows the energy resolution degradation for different crystals, measured as FWHM (\%) of the $511 \mathrm{keV}$ photopeak, after the detectors are recalibrated in the presence of the magnetic field. The recalibration follows the same protocol that a standard calibration: field-flood illuminations are acquired with the tube exposed to the magnetic field, and from them the crystals, phoswich and energy LUTs are calculated. The plots depict the predicted behavior for the energy resolution: larger magnetic fields produce lower output signals whose lower SNR deteriorates the energy resolution; this effect becomes more evident when the main component of the magnetic field impinges the tube parallel to its longitudinal axis.

\section{Conclusions}

We tested the effect of static magnetic fields on the PS-PMTs DOI PET detectors of the Argus PET scanner. The results show that these detectors can withstand magnetic fields up to $1 \mathrm{mT}$ if the main component of the field is perpendicular to the longitudinal axis of the tube, but do not maintain its performance if the magnetic field orientation is parallel to said axis. In the PET/MR
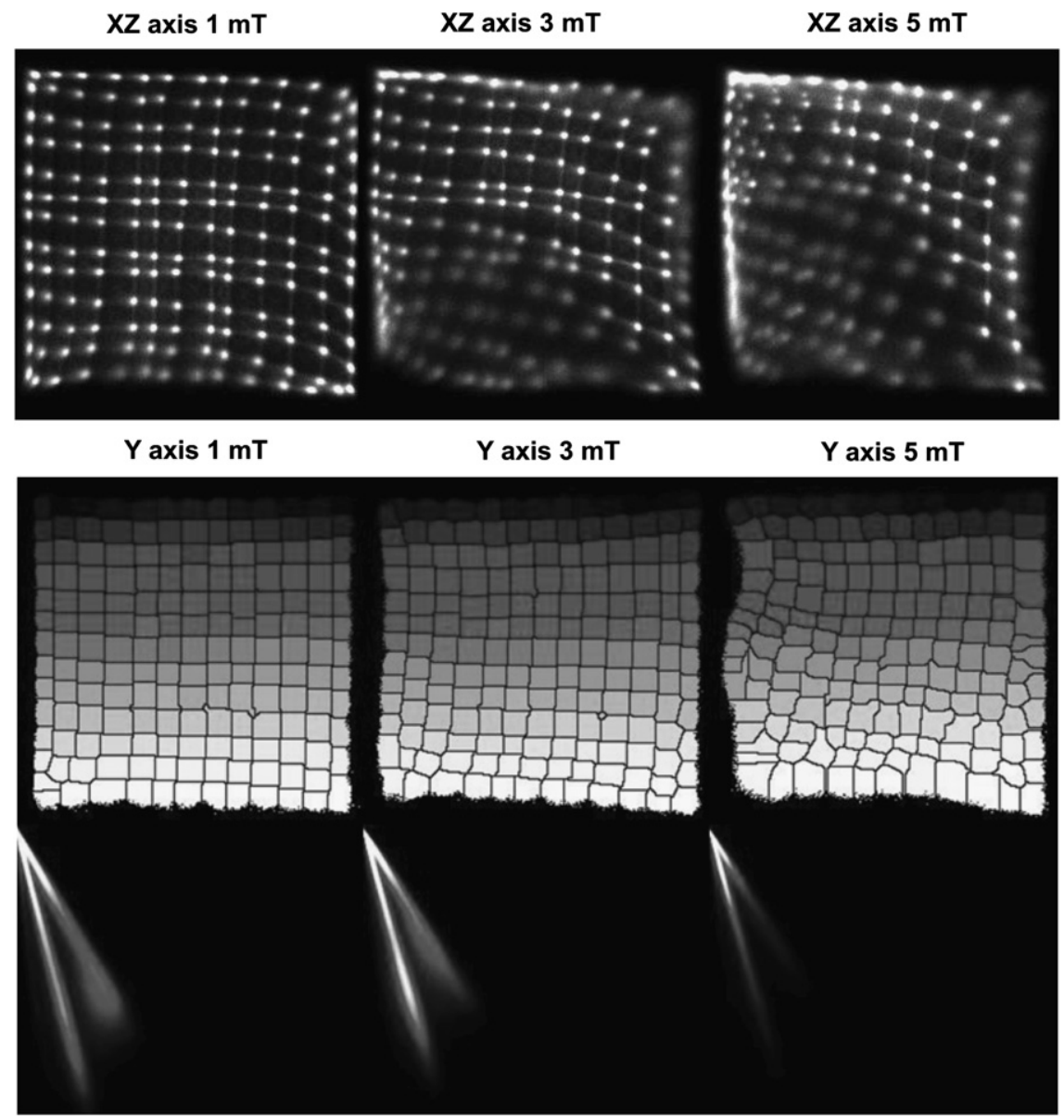

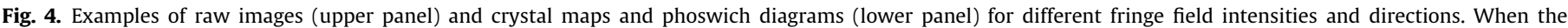

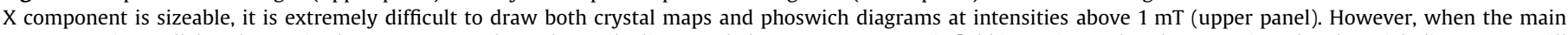

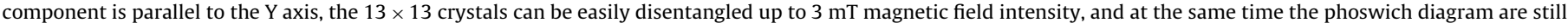
recognizable (lower panel). 

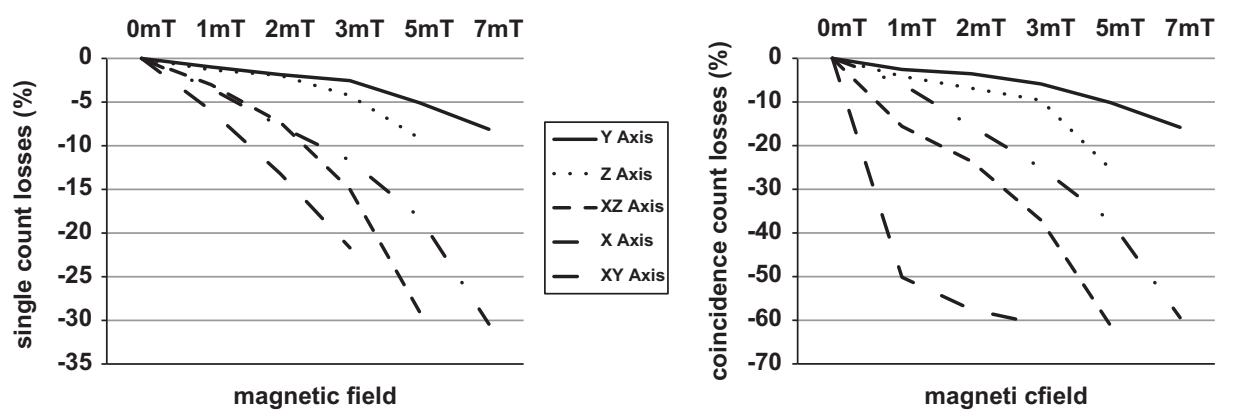

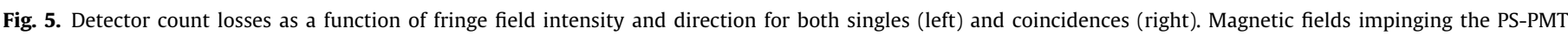
parallel to its longitudinal axis (X axis in out setup) produces the larger losses.
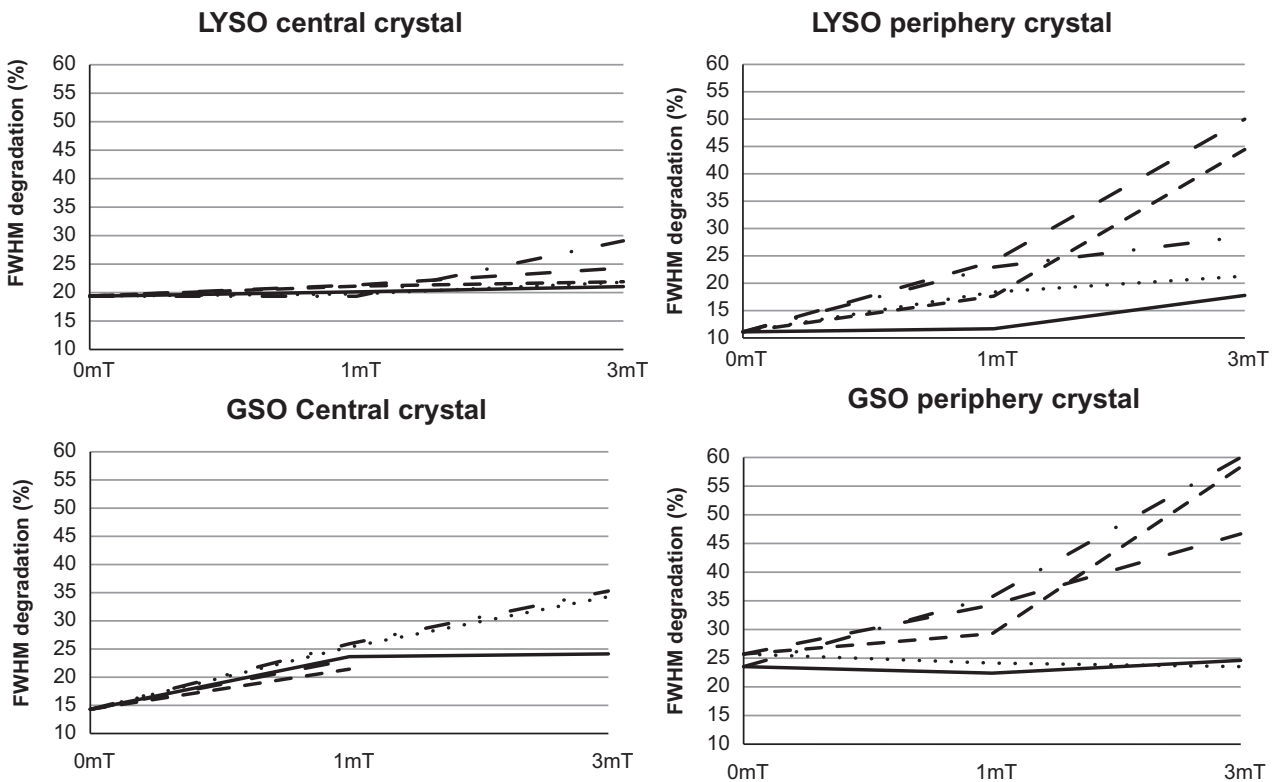

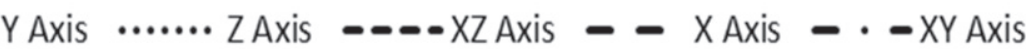

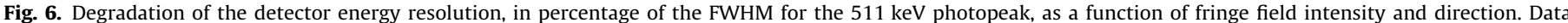

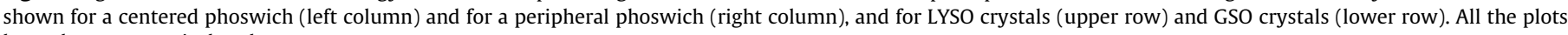
have the same vertical scale.

tandem configuration presented here the parallel component of the magnetic fringe field impinging in the PS-PMT is very low compared with the perpendicular one, in such a way that a recalibration of the system would suffice to keep good image quality.

Therefore, we can conclude that under these conditions (less than $3 \mathrm{mT}$ of perpendicular axis component of the magnetic field, less than $1 \mathrm{mT}$ if a significant parallel component is present), it is still possible to recalibrate the system and to obtain good images. These results encourage us to test the full ring placed at the $1 \mathrm{mT}$ fringe field, replicating the image performance assessment procedure described here.

\section{Acknowledgment}

This work was supported in part by projects CENIT-AMIT Ingenio 2010, TEC2011-28972 (Ministerio de Ciencia e Innovación)
ARTEMIS S2009/DPI-1802 (Comunidad de Madrid, Fondo Social Europeo), and EU IMI Joint Undertaking PreDiCT-TB 115337.

\section{References}

[1] R.R. Raylman, B.E. Hammer, N.L. Christensen, IEEE Transactions on Nuclear Science NS43 (1996) 2406.

[2] Y. Shao, S.R. Cherry, K. Farahani, K. Meadors, S. Siegel, R.W. Silverman, P.K. Marsden, Physics in Medicine and Biology 42 (1997) 1965.

[3] K. Farahani, R. Slates, Y. Shao, R. Silverman, S. Cherry, Journal of Magnetic Resonance Imaging 9 (1999) 497.

[4] R.B. Slates, K. Farahani, Y. Shao, P.K. Marsden, J. Taylor, P.E. Summers, S. Williams, J. Beech, S.R. Cherry, Physics in Medicine and Biology 44 (1999) 2015.

[5] P.K. Marsden, D. Strul, S.F. Keevil, S.C. Williams, D. Cash, British Journal of Radiology 75 Spec no. (2002) S53.

[6] C. Catana, Y. Wu, M.S. Judenhofer, J. Qi, B.J. Pichler, S.R. Cherry, Journal of Nuclear Medicine 47 (2006) 1968.

[7] N. Kawai, K. Miyake, Y. Kuroda, S. Yamashita, Y. Nishiyama, T. Monden, Y. Sasakawa, S. Nagao, Annals of Nuclear Medicine 20 (2006) 371. 
[8] A.J. Lucas, R.C. Hawkes, R.E. Ansorge, G.B. Williams, R.E. Nutt, J.C. Clark T.D. Fryer, T.A. Carpenter, Technology in Cancer Research and Treatment 5 (2006) 337.

[9] B.J. Pichler, M.S. Judenhofer, C. Catana, J.H. Walton, M. Kneilling, R.E. Nutt S.B. Siegel, C.D. Claussen, S.R. Cherry, Journal of Nuclear Medicine 47 (2006) 639

[10] R.R. Raylman, S. Majewski, S.K. Lemieux, S.S. Velan, B. Kross, V. Popov, M.F. Smith, A.G. Weisenberger, C. Zorn, G.D. Marano, Physics in Medicine and Biology 51 (2006) 6371.

[11] M.S. Judenhofer, C. Catana, B.K. Swann, S.B. Siegel, W.I. Jung, R.E. Nutt S.R. Cherry, C.D. Claussen, B.J. Pichler, Radiology 244 (2007) 807.

[12] R.R. Raylman, S. Majewski, S.S. Velan, S. Lemieux, B. Kross, V. Popov, M.F. Smith, A.G. Weisenberger, Journal of Magnetic Resonance 186 (2007) 305

[13] H. Zaidi, O. Mawlawi, C.G. Orton, Medical Physics 34 (2007) 1525

[14] C. Catana, D. Procissi, Y. Wu, M.S. Judenhofer, J. Qi, B.J. Pichler, R.E. Jacobs S.R. Cherry, Proceedings of the National Academy of Sciences of the United States of America 105 (2008) 3705.
[15] M.S. Judenhofer, H.F. Wehrl, D.F. Newport, C. Catana, S.B. Siegel, M. Becker, A. Thielscher, M. Kneilling, M.P. Lichy, M. Eichner, K. Klingel, G. Reischl, S. Widmaier, M. Rocken, R.E. Nutt, H.J. Machulla, K. Uludag, S.R. Cherry, C.D. Claussen, B.J. Pichler, Nature Medicine 14 (2008) 459.

[16] B.J. Pichler, M.S. Judenhofer, C. Pfannenberg, Handbook of Experimental Pharmacology (2008) 109.

[17] H. Zaidi, N. Ojha, M. Morich, Physics in Medicine and Biology 56 (10) (2011) 3091-3106.

[18] Y. Wang, J. Seidel, B.M. Tsui, J.J. Vaquero, M.G. Pomper, Journal of Nuclear Medicine 47 (2006) 1891.

[19] K.K.. Hamamatsu, Photonics, Photomultupliers Tubes: Basics and Applications, 2nd. edition, Hamamatsu Photonics K.K., 1999.

[20] J. Seidel, J.J. Vaquero, F. Barbosa, I.J. Lee, C. Cuevas, M.V. Green, IEEE Transactions on Nuclear Science NS47 (2000) 1640. 